\title{
Rapid Online Identification of Adverse Events After Influenza Immunization in Children by PCIRN's National Ambulatory Network
}

\author{
Julie A. Bettinger, PhD, * Otto G. Vanderkooi, MD, † Judy MacDonald, MD, + and James D. Kellner, MD $†$
}

\begin{abstract}
Background: A National Ambulatory Network was created in 2009 to rapidly assess the safety of influenza vaccines. In 2012, the network was expanded to monitor the safety of live attenuated influenza vaccine and trivalent inactivated influenza vaccine in children.

Methods: We used an online survey administered 7 days after influenza immunization to track new or exacerbated health problems that required medical consultation or prevented daily activities. Parents of immunized children completed the survey. Reported adverse events were followed up by telephone within 48 hours of the online report. A sample of nonresponders was contacted by telephone to ensure the online responder group was representative. Event rates after the 2 influenza vaccines were compared.

Results: A total of 1230 parents completed an online or telephone survey, for a participation rate of $83 \%: 72 \%$ responded online and an additional $11 \%$ were reached by telephone. The rate of severe events in children immunized with an influenza vaccine was $4.7 \%$ (3.5-5.9\%). The frequency and types of events reported were similar between online and telephone reports. Reported rates of severe events were similar after trivalent inactivated influenza or live attenuated influenza vaccine ( $4.0 \%$ vs. $5.1 \%$, respectively). The online survey was easy to access and understand. Most respondents (94\%) would participate next year.

Conclusions: The rate and type of adverse events after immunization with trivalent inactivated influenza versus live attenuated influenza vaccine were similar and did not vary by reporting process (online vs. telephone). The electronic surveillance methodology provided rapid vaccine safety data in children. The electronic survey methodology was acceptable and feasible.
\end{abstract}

Key Words: vaccine safety, pediatric vaccination, surveillance, live attenuated influenza vaccine, internet.

(Pediatr Infect Dis J 2014;33:1060-1064)

B efore the seasonal influenza vaccine is available for use in Canada each year, manufacturers have been required to conduct trials in a limited number $(<200)$ of healthy adults, although these

Accepted for publication April 9, 2014.

From the *Vaccine Evaluation Center, BC Children's Hospital, University of British Columbia, Vancouver, British Columbia; †Alberta Children’s Hospital, University of Calgary, Calgary, Alberta; and $\ddagger$ Alberta Health Services, Calgary Zone, Calgary, Alberta, Canada.

O.G.V. has received research funding from Pfizer Canada Inc. (grant-in-aid), GlaxoSmithKline (contract research), Sanofi Canada (via Canadian Center for Vaccinology; investigator initiated research), Merck Canada (grant-in-aid) and Wyeth Pharmaceuticals (now Pfizer; grant-in-aid). Speaker/discussant at advisory/expert panel meetings for Novartis Canada and Pfizer Canada Inc. J.D.K. has received research funding from Pfizer Canada (investigator initiated grant in aid) and has participated in an expert panel for Pfizer Global.

The Public Health Agency of Canada and the Canadian Institutes of Health Research provided the funding for this study. The funders had no role in this study. J.A.B. is supported by a Career Investigator Award from the Michael Smith Foundation for Health Research.

The authors have no other funding or conflicts of interest to disclose.

Address for correspondence: Dr. Julie Bettinger, BC Children's and Women's Hospital, University of British Columbia, 950 West 28th Avenue, Room A-5, Vancouver, BC V5Z4H4. E-mail: jbettinger@cfri.ca.

Copyright (C) 2014 by Lippincott Williams \& Wilkins

ISSN: 0891-3668/14/3310-1060

DOI: $10.1097 /$ INF.0000000000000373 trials do not have to include Canadians. Preseason trials of annual influenza vaccines rarely involve healthy children and never involve children with underlying health conditions (ie, those most indicated for immunization). Thus most influenza vaccine safety monitoring involves post-marketing passive surveillance.

Vaccine safety monitoring in Canada involves both passive and active surveillance systems. ${ }^{1}$ Passive surveillance captures all spontaneous reports submitted by health care providers or vaccine manufacturers to public health authorities, whereas active surveillance for severe adverse events (AEs) after childhood immunizations is conducted by the Canadian Immunization Monitoring Program, Active. ${ }^{2,3}$ Although passive and active surveillance for vaccine safety are important, both methods have shortcomings for monitoring the safety of influenza vaccines. Passive surveillance suffers from underreporting and reporting bias. Neither type of surveillance permits the calculation of population-based incidence rates of AEs. Importantly, neither method provides sufficiently timely information given that $>100,000$ influenza vaccine doses are administered in the first 4-6 weeks of the annual campaign. ${ }^{1,4-6}$ Delayed recognition of a safety problem means that many vaccinees will already have been affected, potentially harming public confidence in the program.

The need for more rapid and representative safety assessments of seasonal influenza vaccines in children was highlighted in 2010 when an Australian influenza vaccine was associated with an increased rate of fever and convulsions in children..$^{7,8}$ Although Australia's existing vaccine monitoring systems performed well and the safety signal was detected and investigated within 6 weeks of the campaign start, 19,000 doses had been delivered by the time the immunization campaign was suspended. ${ }^{9,10}$ In Canada, $>100,000$ people are vaccinated daily during the first 6 weeks of the influenza immunization campaign, so a similar interval to identify and investigate a safety signal could represent millions of doses distributed. ${ }^{11}$

Recognizing the need for improved, rapid safety surveillance for influenza vaccines, particularly in children, the Public Health Agency of Canada/Canadian Institutes of Health Research Influenza Research Network's National Ambulatory Network was created to quickly gather and analyze safety data on thousands of vaccinated and unvaccinated individuals. The objective is to provide real-time safety information to public health authorities before the core weeks of the annual influenza immunization campaign. Since 2009, the network has used online surveys to monitor selected adverse events after influenza immunization of over 10,000 healthcare workers annually. ${ }^{12}$

As a pilot project in the fall of 2012, the catchment expanded to include children in a jurisdiction using the live attenuated intranasal influenza vaccine (LAIV) for the first time. The main outcome was a severe AE after immunization, which was defined as the occurrence of a new health event or the worsening of an existing health problem severe enough to cause medical consultation and/or prevent daily activities. However, the survey also collected data on milder outcomes that are relevant for safety monitoring and important to parents. This report describes the characteristics of 
the reporting system, the outcomes of the first year of surveillance in children and the acceptability of electronic reporting among parent participants.

\section{MATERIALS AND METHODS}

A convenience sample of parents of children 6 months to 18 years of age whose child(ren) received a 2012 influenza vaccine (FluMist, MedImmune Vaccines, Inc, Philadelphia, PA; Agriflu, Novartis Canada, Montreal, Canada or Fluviral, GlaxoSmithKline, Mississauga, Canada) from mass immunization clinics in Calgary, Alberta, were recruited from October 15 to 27, 2012. The mass venue clinics were open to all members of the public and no appointments were taken. Within these influenza vaccine clinics, study staff approached the parents or caregivers of children in the waiting rooms to ask their consideration for participation. No record was kept of the total number of children approached. Participants were recruited before immunization as they waited or during the 15 minute anaphylaxis observation time afterward. Only participants with an active e-mail address and telephone were recruited. Children were eligible to participate after the first or second dose of influenza vaccine. At enrollment, all participants provided their e-mail address, telephone number(s) and written informed consent. The study was approved by research ethics boards at the University of British Columba and the University of Calgary.

\section{Study Procedures}

Participants were sent an e-mail at 8 days after immunization inviting them to respond to a short online survey. Parents completed separate reports for each immunized child. Nonresponders were sent a reminder e-mail with the survey link 3 days later (11 days postimmunization). A sample of participants who did not respond after the reminder e-mail were contacted by telephone 1517 days postimmunization (5-7 days after the reminder e-mail) to allow comparison of event rates and characteristics between online and telephone responders.

The main outcome was a severe AE after immunization, which was defined as the occurrence of a new health event or the worsening of an existing health problem severe enough to cause medical consultation and/or prevent daily activities. Those who reported a severe health event meeting the above criteria on the web survey were contacted by telephone within 48 hours by a nurse specifically trained in obtaining a detailed history of possible AEs after immunization. This allowed for validation of the severe event reported online, measurement of agreement between the online reporting and telephone follow up and for the collection of additional details about the event (ie, symptoms, diagnosis, duration, treatment and outcome). The additional event details were entered into an electronic AE report form. Any eligible AEs were reported to public health.

A secure online survey and AE report form were created (SimpleSurvey v2.17.0, OutSideSoft Solutions inc., Saint-Jeansur-Richelieu, Quebec). The survey captured AEs as noticed by the parents without any specific monitoring instructions. For example, parents were not instructed to measure fever. The survey collected information on the following: demographics (age of child, sex and any underlying health conditions), past influenza immunization history of the child and the occurrence of any AE during the first 7 days postimmunization. Preferred method of response (online survey vs. text messaging vs. telephone, etc.) and questions to assess the ease and usability of the AE self-reporting process and survey were asked at the end of the questionnaire. Answers were primarily binary (ie, In the first week (7 days) after vaccination did your child have any new symptoms severe enough to limit his/her normal daily activities? Yes or no) with categorical questions used to measure severity of symptoms, devices used for method of response and ease and usability of the process. Open text fields described other AEs, comments, suggested changes to the survey or process. Telephone follow up of the nonresponders captured the same information as well as the reason for nonresponse online. The symptoms selected for monitoring were based on provincial and national guidelines for reporting adverse events following immunization. ${ }^{13,14}$ The online survey has been included as supplementary material (see parental survey, Supplemental Digital Content 1, http://links.lww.com/INF/B897).

\section{Analysis}

Participants were identified by a unique study code and e-mail addresses were not linked with the survey responses. For the analysis, participants were categorized into online (this group responded to the survey online) and telephone responders (this group completed the survey by telephone) and by vaccine type [LAIV vs. trivalent inactivated influenza (TIV)]. The characteristics of the responders, the types and severity of reported AE and participant opinions on the ease and usability of the survey were summarized using SAS version 9.3 (SAS Institute, Cary, NC). AE were classified into broad system categories and $95 \%$ confidence intervals were calculated for the rate of events overall, in each respondent group and by vaccine type. ${ }^{15}$ We calculated a sample of 1000 respondents would provide $90 \%$ probability to detect events occurring at a rate of 1 in $500(0.002)$.

\section{RESULTS}

A total of 1489 children enrolled in the study with $83 \%$ ( $n=$ $1230)$ completing the survey: $1070(72 \%)$ responded to the online survey and an additional $160(11 \%)$ were reached by telephone. Close to $40 \%(160 / 419)$ of the nonresponders to the online survey were contacted by telephone. Seventy-six percent of respondents had completed the survey by November 5, 3 weeks after the annual campaign started. The last AE follow-up interview was completed November 21. The children of online and telephone responders were similar in their demographic characteristics (Table 1). An equal proportion of children with underlying health conditions received TIV and LAIV (50\% for each vaccine). The majority of healthy children received LAIV (69\%).

The type and frequency of AEs reported online by respondent type and vaccine are shown in Table 2 . The reported rate of wheezing was not significantly different by vaccine $(0.2 \%$ LAIV vs. $0.5 \%$ TIV, $P=0.47$ ). Of the 99 events reported, 5 were easily tolerable, 27 were uncomfortable and the remaining 65 met the criteria for severe. No neurologic symptoms were reported online or in the subsequent follow up of severe events. The severe event reporting rate in online respondents was $5.1 \%$ (95\% confidence interval: $3.8-6.5 ; \mathrm{n}=55)$ compared with a rate of $6.3 \%(2.5 \%-10.0 \%)$ in telephone respondents $(n=10)$. Four respondents reported an event severe enough to cause them to seek medical care, 55 reported an event severe enough to prevent daily activities and 6 reported an event severe enough to prevent daily activities and to cause them to seek medical care. The overall frequency of severe events did not vary by age group (data not shown).

Sixty-three severe event reports were followed up by telephone ( 2 participants were not reached) and 58 were true events ( 5 of the severe events reported online were reporting errors). Thus the validated event rate was $4.7 \%(3.5 \%-5.9 \%)$. The validated severe event rates by vaccine were similar: LAIV 5.1\% (3.7-6.9) and TIV $4.0 \%$ (2.3-6.4). Details of the severe AE by vaccine type as reported on follow up are shown in Figure 1. The majority of events reported $(n=44)$ lasted $>24$ hours for an average duration of 2.8 days (range 1.0-7.0 days). For events that resolved within 24 hours, the average time was 9 hours (range 4.0-18.0 hours). 
TABLE 1. Characteristics of Vaccinated Children

\begin{tabular}{|c|c|c|c|c|c|c|}
\hline & \multicolumn{2}{|c|}{$\begin{array}{l}\text { Online Responder } \\
(\mathrm{n}=\mathbf{1 0 7 0})\end{array}$} & \multicolumn{2}{|c|}{$\begin{array}{l}\text { Telephone Responder } \\
\text { (n = 160) }\end{array}$} & \multicolumn{2}{|c|}{$\begin{array}{c}\text { Total } \\
(\mathbf{N}=1230)\end{array}$} \\
\hline & $\mathbf{N}$ & $(\%)$ & $\mathbf{N}$ & $(\%)$ & $\mathbf{N}$ & $(\%)$ \\
\hline Female & 524 & $(49.0)$ & 79 & $(49.4)$ & 603 & $(49.0)$ \\
\hline \multicolumn{7}{|l|}{ Age } \\
\hline 6-23 months & 83 & (7.8) & 17 & $(10.6)$ & 100 & $(8.1)$ \\
\hline $2-4$ years & 253 & $(23.6)$ & 27 & (16.9) & 280 & $(22.8)$ \\
\hline $5-9$ years & 445 & $(41.6)$ & 68 & $(42.5)$ & 513 & (41.7) \\
\hline 10-17 years & 289 & $(27.0)$ & 48 & $(30.0)$ & 337 & $(27.4)$ \\
\hline Underlying health condition & 129 & (12.1) & 25 & (15.6) & 154 & (12.5) \\
\hline Previous influenza vaccine & 944 & $(88.2)$ & 146 & $(91.3)$ & 1090 & $(88.6)$ \\
\hline \multicolumn{7}{|l|}{ Vaccine received } \\
\hline LAIV & 714 & $(66.7)$ & 102 & $(63.8)$ & 816 & $(66.3)$ \\
\hline TIV & 347 & $(32.4)$ & 56 & $(35.0)$ & 403 & $(32.8)$ \\
\hline Do not know & 9 & $(0.8)$ & 2 & (1.3) & 11 & $(0.9)$ \\
\hline
\end{tabular}

Note: no significant difference between online and telephone responder characteristics.

In terms of outcome, $78 \%(45 / 58)$ of severe events had resolved by the time of the telephone follow up (within 48 hours of reporting), $21 \%(12 / 58)$ were improving and $1.7 \%$ (1/58) remained unchanged.

Of the 10 participants who reported events severe enough for medical care in the online survey, only 7 actually sought care. An additional 3 respondents who reported events severe enough to prevent daily activities had sought care by the time of their followup phone call. For the 10 individuals who sought care, 1 sought telephone advice for wheezing symptoms 6 hours after immunization with TIV, 7 were seen in a clinic setting and 2 were seen in a hospital emergency department ( 1 for an allergic reaction 2 hours after immunization with TIV and 1 for anaphylaxis thought to be induced by exercise 1 day after immunization with LAIV).

\section{Survey Characteristics}

The error rate for severe events in the online reporting was $0.5 \%(5 / 1070)$. The percentage of agreement between severe events reported online and the subsequent telephone interview was high for allergic events $(93 \%)$, respiratory symptoms/infections (cold, sore throat; 84\%) and gastrointestinal symptoms (79\%) and lower for systemic symptoms (fever, irritability, anorexia; 67\%).
The survey was rated easy to access by $98 \%$ of the online respondents and $33 \%$ of the telephone respondents. It was easy to understand for $99 \%$ of online respondents and $88 \%$ of telephone responders and $94 \%$ of online and $97 \%$ of telephone respondents would complete the survey next year. Among those who required the e-mail reminder, 91\% found it helpful. Most online respondents $(76 \%)$ used a computer to complete the survey, followed by a mobile phone $(12 \%)$ or tablet $(10 \%)$. Most online and telephone responders $(96 \%)$ preferred to receive the invitation for the survey through e-mail and $98 \%$ of online responders and $89 \%$ of telephone responders preferred to complete the survey online with almost $9 \%$ of telephone responders $(n=14)$ preferring to complete the survey over the phone. The main reasons telephone responders gave for not completing the survey online was being too busy/forgot $(47 \%$, $\mathrm{n}=75$ ) or not receiving the survey invitation/being unable to open it $(38 \%, \mathrm{n}=61)$.

The personnel resources required for study implementation were as follows: a study coordinator or manager for 3 months at halftime or approximately 15 hours a week and a research assistant for 3 months for 12 hours a week; recruitment required 2 individuals full-time for 2 weeks; nursing time for both the AE follow up and nonresponder telephone calls was 55 hours over 4 weeks.

TABLE 2. Type and Frequency of Any AE After Influenza Immunization in Children Reported 7 Days After Immunization

\begin{tabular}{|c|c|c|c|c|c|c|c|c|c|c|}
\hline \multirow[b]{3}{*}{$\mathrm{AE}$} & \multicolumn{4}{|c|}{ Respondent Type } & \multicolumn{4}{|c|}{ Vaccine Type } & \multicolumn{2}{|c|}{ Overall } \\
\hline & \multicolumn{2}{|c|}{$\begin{array}{c}\text { Online } \\
(\mathrm{N}=1070)\end{array}$} & \multicolumn{2}{|c|}{$\begin{array}{l}\text { Telephone } \\
(\mathrm{N}=160)\end{array}$} & \multicolumn{2}{|c|}{$\begin{array}{c}\text { LAIV } \\
(\mathrm{N}=816)\end{array}$} & \multicolumn{2}{|c|}{$\begin{array}{c}\text { TIV } \\
(\mathrm{N}=403)\end{array}$} & \multicolumn{2}{|c|}{$(\mathrm{N}=1230)$} \\
\hline & $\mathrm{N}$ & $(\%)$ & $\mathrm{N}$ & $(\%)$ & $\mathrm{N}$ & $(\%)$ & $\mathrm{N}$ & $(\%)$ & $\mathrm{N}$ & $(\%)$ \\
\hline Any event & 89 & $(8.3)$ & 10 & $(6.3)$ & 73 & $(8.9)$ & 26 & $(6.5)$ & 99 & $(8.0)$ \\
\hline Fever $>38.5$ & 22 & $(2.1)$ & 6 & $(3.7)$ & 18 & $(2.2)$ & 10 & $(2.5)$ & 28 & $(2.3)$ \\
\hline Fever/chills & 43 & $(4.0)$ & 4 & $(2.5)$ & 37 & $(4.5)$ & 10 & $(2.5)$ & 47 & (3.9) \\
\hline Anorexia & 30 & $(2.8)$ & 5 & $(3.1)$ & 25 & $(3.1)$ & 10 & $(2.5)$ & 35 & $(2.9)$ \\
\hline Persistent crying & 0 & $(0.0)$ & 0 & $(0.0)$ & 0 & $(0.0)$ & 0 & $(0.0)$ & 0 & $(0.0)$ \\
\hline Seizure/convulsion & 0 & $(0.0)$ & 0 & $(0.0)$ & 0 & $(0.0)$ & 0 & $(0.0)$ & 0 & $(0.0)$ \\
\hline Wheezing & 4 & $(0.4)$ & 0 & $(0.0)$ & 2 & $(0.2)$ & 2 & $(0.5)$ & 4 & $(0.3)$ \\
\hline Rash/itching/tingling skin & 5 & $(0.5)$ & 0 & $(0.0)$ & 2 & $(0.2)$ & 3 & $(0.7)$ & 5 & $(0.4)$ \\
\hline $\begin{array}{l}\text { Respiratory infection symptoms } \\
\text { (cold, sore throat) }\end{array}$ & 56 & $(5.2)$ & 3 & (1.9) & 47 & $(5.8)$ & 12 & $(3.0)$ & 59 & (4.8) \\
\hline Nausea, vomiting, diarrhea & 33 & $(3.0)$ & 5 & $(3.1)$ & 30 & $(3.7)$ & 8 & $(2.0)$ & 38 & (3.1) \\
\hline Other* & 20 & (1.9) & 2 & (1.3) & 18 & $(2.2)$ & 4 & $(1.0)$ & 22 & (1.8) \\
\hline
\end{tabular}

*Other included: headache, irritability/tiredness, ear infection, decreased eating and anaphylaxis. 


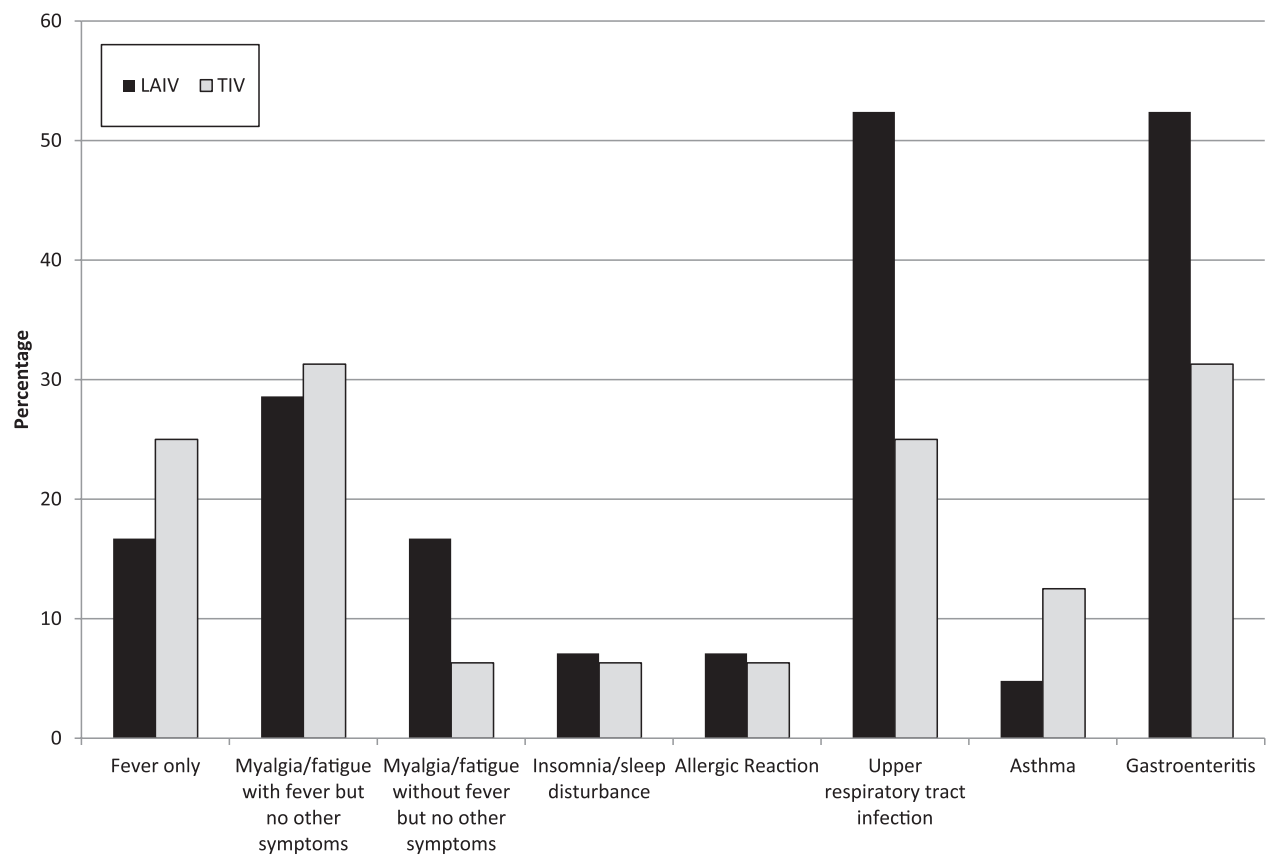

FIGURE 1. Severe events by LAIV and TIV in children 6 months to 17 years of age as reported on follow-up interview. Note: No significant differences were found in severe events reported by LAIV and TIV vaccinees.

Development of the online survey, data management and analysis required an additional 3 weeks of full-time work for 2 individuals.

\section{DISCUSSION}

Our study provides the first assessment of online, rapid safety surveillance of seasonal pediatric influenza immunizations. Moreover, it provided rapid data on AE occurring in children early in the influenza immunization campaign. Reassuringly, we found only minor differences between the AE reported after TIV and LAIV in our population indicating a good safety profile for both vaccines in our study respondents. The rate of wheezing did not differ by vaccine type. Respiratory infection, fever/chills and gastrointestinal symptoms were the most frequent severe health problems reported regardless of vaccine type.

Not surprisingly, because of the solicited nature of $\mathrm{AE}$ reporting in clinical trials, the rates for most postvaccination events (eg, fever, respiratory infections, gastroenteritis and wheezing) were higher in influenza clinical trial data for both LAIV and TIV than in our study. For instance, $10-35 \%$ of participants in LAIV and TIV clinical trials reported fever ${ }^{16-18}$ whereas our rates were $\leq 3 \%$ after both vaccines. Our results were closer to reports from Canadian passive surveillance that found TIV postvaccination fever in $1-5 \%$ of children $1-5$ years of age. ${ }^{19}$

In terms of serious events for LAIV, we identified more respiratory infections, gastroenteritis and systemic events than those reported to the Vaccine Adverse Event Reporting System (VAERS). ${ }^{20}$ We would expect this, given the differences in the definition of serious events between VAERS and our study. Serious reports in VAERS are defined as death, life-threatening conditions, hospitalization, disability or congenital anomaly, ${ }^{20}$ whereas our definition was designed to be less stringent to elicit potential signals. Interestingly, the proportion of serious allergic reactions (excluding anaphylaxis) after LAIV reported in our study (7.1\%) was similar to VAERS $(7.5 \%){ }^{20}$ This similarity may reflect the fact that these events in our study resulted in medical consultations and thus more closely reflected the VAERS definition for serious events.
Severe AE rates of 5.1\% (95\% confidence interval: $3.8-$ $6.5)$ for online respondents and $6.3 \%(2.5-10.0 \%)$ for telephone respondents showed no significant differences by response method. However, our study was only powered to detect a difference of $6 \%$ in reporting rates between the 2 groups, so smaller differences may exist. Our results indicate large differences did not occur. Importantly, participants did not appear more likely to complete the online survey if they experienced an event. The subsequent telephone follow up of the severe events showed high agreement with what was reported online, with the more specific events (ie, nausea, allergic reactions), showing higher agreement than nonspecific events such as feverishness or irritability.

One case of anaphylaxis was reported in the "other" category online. This serves to highlight the importance of a general catch-all category and short text fields to capture a description of unexpected $\mathrm{AE}$ and to allow parents a way to specifically report severe AE that may not fit into the survey's designated categories. It also illustrated a need to include an anaphylaxis category in our survey for next year as this is an event we would like to capture systematically.

Although a number of studies have used internet-based reporting to examined vaccine safety in adults, ${ }^{4,21-23}$ our survey is the first, to our knowledge, to examine seasonal influenza vaccine safety among children in real-time using online parental reporting. Real-time data mining of electronic medical records as occurs in the United States with the Vaccine Safety Datalink ${ }^{24}$ is not yet feasible in Canada. Not all Canadian jurisdictions collect individual-level influenza immunization data electronically, medical and immunization records are rarely linked and the barriers for such linkages can be great. ${ }^{25-29}$ Therefore, the methodology employed in this study can be used to fill an important gap in Canadian vaccine safety surveillance.

As a pilot study, the enrollment was purposefully low. Unfortunately, this did not allow us to detect differences in severe event symptoms between the 2 vaccine types or to detect rare $\mathrm{AE}$. In the future, enrolling approximately 10,000 children, as we do with our health care worker surveillance, ${ }^{12}$ would allow us to detect $\mathrm{AE}$ occurring at a rate of about $1 / 1000$, which is high enough to 
detect anomalies such as the 2010 seizure clusters in Australia ${ }^{8,30}$ and to provide information on the emerging safety profile of the seasonal influenza vaccines in children. It could also highlight any differences in severe event symptoms by vaccine type.

One of the shortcomings of the pilot study was our inability to determine the background rates for common events (eg, colds, fever) in our population. Therefore, in fall 2013, we will enroll a not-yet immunized group of children for a 7-day health event survey, thus enabling us to compare event rates pre- and postimmunization. We also did not track the total number of parents approached to take part in the study. However, in discussion with the study staff, their recollection was that a high percentage of those approached agreed to participate, likely $80 \%$ or higher. Finally, we expect that written informed consent will not be necessary if the program is expanded. To determine this, we will be pilot testing online selfregistration and online consent in fall 2013.

\section{CONCLUSIONS}

Both the LAIV and TIV vaccines had a similarly good safety profile after administration to over 1200 children. Notably, the rate of wheezing was not different between recipients of the 2 vaccines. The results from this initial pilot demonstrate internet-based safety surveys administered to parents with telephone follow up for severe events is highly acceptable and feasible and can successfully be used in the pediatric population to provide rapid safety data to decision makers. This methodology provides an important complement to existing Canadian surveillance to monitor the safety of seasonal influenza vaccines in children and it may work well in other countries with high internet penetration.

\section{ACKNOWLEDGMENTS}

The authors gratefully acknowledge the expert assistance provided by the Vaccine Evaluation Center (Judy Needham, Kim Marty and Wenli Zhang).

Authors' contribution: J.A.B. was involved in the conception and design of the study, the analysis and interpretation of the data and wrote the article. O.G.V. was involved in design of the study, the acquisition of the data and revision of the manuscript. J.M. was involved in the acquisition of data and revision of the manuscript. J.D.K. was involved in the design of the study, acquisition of the data and revision of the manuscript.

\section{REFERENCES}

1. Part 2 Vaccine Safety and Adverse Events Following Immunization. Canadian Immunization Guide. Evergreen Edition ed, Public Health Agency of Canada: Ottawa, Ontario, Canada; 2012.

2. Scheifele DW. IMPACT after 17 years: lessons learned about successful networking. Paediatr Child Health. 2009;14:33-35.

3. Scheifele DW, Halperin SA; CPS/Health Canada, Immunization Monitoring Program, Active (IMPACT). Immunization Monitoring Program, Active: a model of active surveillance of vaccine safety. Semin Pediatr Infect Dis. $2003 ; 14: 213-219$.

4. Newes-Adeyi G, Greece J, Bozeman S, et al. Active surveillance for influenza vaccine adverse events: the integrated vaccine surveillance system. Vaccine. 2012;30:1050-1055.

5. Gold MS, Effler P, Kelly H, et al. Febrile convulsions after 2010 seasonal trivalent influenza vaccine: implications for vaccine safety surveillance in Australia. Med J Aust. 2010;193:492-493.

6. Iskander J, Broder K. Monitoring the safety of annual and pandemic influenza vaccines: lessons from the US experience. Expert Rev Vaccines. 2008;7:75-82.

7. Petousis-Harris H, Poole T, Booy R, et al. Fever following administration of two inactivated influenza vaccines-a survey of parents of New Zealand infants and children 5 years of age and under. Vaccine. 2011;29:2933-2937.

8. ATAGI Statement: Clinical Advice for Immunisation Providers on Resumption of the Use of 2010 Trivalent Seasonal Vaccines in Children Less Than 5 Years of Age. Australian Government Department of Health and Aging: Canberra, Australia; 2010.

9. Wood N, Sheppeard V, Cashman P, et al. Influenza vaccine safety in children less than 5 years old: the 2010 and 2011 experience in Australia. Pediatr Infect Dis J. 2012;31:199-202.

10. Overview of Vaccine Regulation and Safety Monitoring and Investigation Into Adverse Events Following 2010 Seasonal Influenza Vaccination in Young Children. Government of Australia Department of Health and Aging: Canberra, Australia; 2010.

11. Kendal AP, MacDonald NE. Influenza pandemic planning and performance in Canada, 2009. Can J Public Health. 2010;101:447-453.

12. De Serres G, Gariépy MC, Coleman B, et al.; PHAC-CIHR influenza Research Network (PCIRN). Short and long-term safety of the 2009 AS03adjuvanted pandemic vaccine. PLoS One. 2012;7:e38563.

13. Adverse events following immunzation reporting 2013-10-03. Available at: http://www.phac-aspc.gc.ca/im/aefi-essi-form-eng.php. Accessed February $22,2014$.

14. Adveres events following immunization forms 2014-01-03. Available at: http://www.bccdc.ca/imm-vac/ForHealthProfessionals/AdverseEventsImms. htm. Accessed February 22, 2014.

15. Armitage P, Berry G, Mathews J. Statistical Methods in Medical Research. 4th ed. Oxford: Blackwell Science; 2001.

16. Ambrose CS, Yi T, Falloon J. An integrated, multistudy analysis of the safety of Ann Arbor strain live attenuated influenza vaccine in children aged 2-17 years. Influenza Other Respir Viruses. 2011;5:389-397.

17. Neuzil KM, Dupont WD, Wright PF, et al. Efficacy of inactivated and coldadapted vaccines against influenza A infection, 1985 to 1990: the pediatric experience. Pediatr Infect Dis J. 2001;20:733-740.

18. Nolan T, Richmond PC, McVernon J, et al. Safety and immunogenicity of an inactivated thimerosal-free influenza vaccine in infants and children. Influenza Other Respir Viruses. 2009;3:315-325.

19. Bexsero: EPAR - product information 03/01/2014 2013. Available at: http://www.ema.europa.eu/ema/index.jsp?curl=pages/medicines/human/ medicines/002333/human_med_001614.jsp\&mid=WC0b01ac058001d124. Accessed February 6, $201 \overline{4}$.

20. Izurieta HS, Haber P, Wise RP, et al. Adverse events reported following live, cold-adapted, intranasal influenza vaccine. JAMA. 2005;294:2720-2725.

21. Mackenzie IS, MacDonald TM, Shakir S, et al. Influenza H1N1 (swine flu) vaccination: a safety surveillance feasibility study using self-reporting of serious adverse events and pregnancy outcomes. Br J Clin Pharmacol. 2012;73:801-811.

22. Lapphra K, Dobson S, Bettinger JA. Acceptability of Internet adverse event self-reporting for pandemic and seasonal influenza immunization among health care workers. Vaccine. 2010;28:6199-6202.

23. Härmark L, van Hunsel F, Hak E, et al. Monitoring the safety of influenza A (H1N1) vaccine using web-based intensive monitoring. Vaccine. 2011;29:1941-1947.

24. Baggs J, Gee J, Lewis E, et al. The Vaccine Safety Datalink: a model for monitoring immunization safety. Pediatrics. 2011;127(suppl 1):S45-S53.

25. Heidebrecht CL, Pereira JA, Quach S, et al.; Public Health Agency of Canada/Canadian Institutes of Health Research Influenza Research Network (PCIRN) Vaccine Coverage Theme Group. Approaches to immunization data collection employed across Canada during the pandemic (H1N1) 2009 influenza vaccination campaign. Can J Public Health. 2011;102:349-354.

26. Pereira JA, Heidebrecht CL, Quach S, et al.; Public Health Agency of Canada/Canadian Institutes of Health Research Influenza Research Network (PCIRN) Vaccine Coverage Theme Group. Influenza immunization data: can we make order out of chaos? Healthc Q. 2011;14:6-7.

27. Why collect individual-level vaccination data? CMAJ. 2010;182:273-275.

28. Kwong JC, Foisy J, Quan S, et al.; Pubic Health Agency of Canada/ Canadian Institutes of Health Research Influenza Research Network (PCIRN). ICES reports: Canada's response to pandemic H1N1 influenza: the collection of individual-level data at the point of vaccination. Healthc Q. 2010;13:18-20.

29. Scheifele DW, Naus M, Crowcroft NS, et al. Optimizing Canadian public immunization programs: a prescription for action. Can J Public Health. 2011;102:193-195.

30. Armstrong PK, Dowse GK, Effler PV, et al. Epidemiological study of severe febrile reactions in young children in Western Australia caused by a 2010 trivalent inactivated influenza vaccine. BMJ Open. 2011;1:e00016. 